\title{
SEMANTICS FOR LOGIC PROGRAMS WITHOUT OCCUR CHECK
}

\author{
W. P. WEIJLAND \\ Centre for Mathematics and Computer Science, Kruislaan 413, 1098 SJ Amsterdam, The Netherlands
}

\begin{abstract}
For reasons of efficiency, in almost all implementations of Prolog the occur check is left out. This a mechanism should protect the program against introducing circular bindings of variables. In practice the occur check is very expensive, however, and it is left to the skills of the user, to avoid these circular bindings in the program. In this paper a semantics of Prolog without occur check is introduced. The new kind of resolution, i.e. SLD-resolution without occur check, is referred to as CSLD-resolution. Important theorems such as soundness and completeness of both CSLD-resolution and the "negation as failure" rule, are established.
\end{abstract}

\section{Introduction}

For reasons of efficiency, in almost all implementations of Prolog the occur check is left out, which is a mechanism that should protect the program against introducing circular bindings of variables. For instance in a substitution $\{x / f(x)\}$, the variable $x$ is bound to a term $f(x)$ containing the variable $x$ again. The problem is, that any such binding endangers the correct behaviour of a Prolog system. In fact, without the occur check we no longer have soundness of SLD-resolution (see [12]). For example consider the program

$$
\begin{aligned}
P: \quad & \text { test } \leftarrow p(x, x) \\
& p(y, f(y)) \leftarrow .
\end{aligned}
$$

Given the goal $\leftarrow$ test, a Prolog system without occur check will answer "yes" since $p(x, x)$ will be successfully unified with $p(y, f(y))$ by the substitution $\{x / y, y / f(y)\}$. However, this answer is quite wrong, since test is not a logical consequence of $P$.

In practice, however, the occur check is very expensive and it is usually left to the skills of the user to avoid these circular bindings in the program. For instance in [14], a method is presented to detect circular bindings more efficiently, by preprocessing Prolog programs.

It would be convenient to develop a theory for SLD-resolution without occur check, and for this reason Prolog II (see [3]) has been studied quite intensively in the past few years. Roughly speaking, Prolog II is standard Prolog without occur check and can be regarded as a system which manipulates infinite trees (see [2]).

The question remains, whether or not Prolog II can be thought of as a logic programming language, since the example above shows that Prolog II presents 
incorrect derivations. This problem was solved in [6], by formulating a soundness theorem for Prolog II. In the above example, the computed substitution $\{x / y$, $y / f(y)\}$ can be translated to a set of equations $\{x=y, y=f(y)\}$, and clearly test is a logical consequence of $P \cup\{x=y, y=f(y)\}$. There are still many results left to be established, such as completeness, to develop a complete theory for Prolog II.

In this paper a semantics for logic programs without occur check is presented by considering circular bindings $\{x / f(x)\}$ as recursive equations $\{x=f(x)\}$, and extending the Herbrand universe (consisting of all closed terms) by adding to it all infinite terms $\{x=f(f(f(\ldots)))\}$ (see [4]). We introduce a new kind of resolution, which will be referred to as CSLD-resolution (complete SLD-resolution), which is precisely SLD-resolution but without occur check. Following this idea, we find that both soundness and completeness for CSLD-resolution as well as for the negation as failure rule is obtained. It turns out that due to the new setting, the proof of the completeness theorem for the negation as failure rule becomes much shorter compared with the well-known proofs in [9], [12] and [15]. Then, we conclude as a general result that $\operatorname{comp}(P) \cup\{A\}$ has a "complete" Herbrand model iff it has a model, which indicates that we may expect CSLD-resolution to have some nice extra properties that we do not have for ordinary SLD-resolution.

Independently from this paper, similar results were stated in [7] and [13] on the "Constraint Logic Programming" scheme, in [8] on Prolog II as a Logic Programming Language scheme, and in [10], giving a logical semantics to a language without occur check containing both equations and inequations.

There are a few theoretical differences between these references and the contents of this paper. Consider for example the fact that we will only need a small equational theory for Prolog II, whereas in [6] and [8] this theory contains infinitely many existential formulas, one for every recursive equation. For this reason we do not need to put any constraints on the models of Prolog II programs and the results are more general. Still, apart from the question of whether the main results are new, we believe we have found a rather elegant formalisation of the theory, making the techniques used in this paper of interest by themselves. The concepts and notations in this paper are quite similar to those in [12], which may be considered a contribution to standardising the theory of logic programming without occur check.

\section{Complete Herbrand models}

We will assume $P$ to be a set of program clauses $\forall\left(B_{1} \wedge \cdots \wedge B_{k} \rightarrow A\right)$, usually written as $A \leftarrow B_{1}, \ldots, B_{k}$, where $B_{1}, \ldots, B_{k}, A$ are atoms not containing "=". The language of $P$ will be denoted by $L(P)$ or $L_{P}$. In this section we will formally introduce complete Herbrand models for $P$. First we will present a precise definition of a complete term, as can be found in [12], and next establish some general model theoretical results. 
Let $\omega^{*}$ be the set of all finite sequences of non-negative integers. Such a finite sequence will be written as $\left[i_{1}, \ldots, i_{k}\right]$, for some $i_{1}, \ldots, i_{k} \in \omega$. For all $m, n \in \omega^{*}$ we write $[m, n]$ for the concatenation of $m$ and $n$, and for $i \in \omega$ we write $[m, i]$ instead of $[m[i]]$. For $X \in \omega^{*}$ we write $|X|$ for the cardinality of $X$.

Definition 2.1. $T \subseteq \omega^{*}$ is called a tree if $T$ satisfies the following conditions:

(i) for all $n \in \omega^{*}$ and $i, j \in \omega,[n, i] \in T \wedge j<i \Rightarrow n \in T \wedge[n, j] \in T$

(ii) $|\{i:[n, i] \in T\}|$ is finite for all $n \in T$.

So, by Definition 2.1 we can interpret [] as the root of the tree and $[n, 0]$, $[n, 1], \ldots,[n, k]$ as the descendents of the node $n$ for all $n \in T, k<\omega$. Now let $S$ be a set of symbols and ar: $S \rightarrow \omega$ be a mapping defining the arity of a symbol.

Definition 2.2. A complete term (over $S$ ) is a function $t: \operatorname{dom}(t) \rightarrow S$ such that:

(i) the domain of $t, \operatorname{dom}(t)$, is a non-empty tree

(ii) for all $n \in \operatorname{dom}(t), \operatorname{ar}(t(n))=|\{i:[n, i] \in \operatorname{dom}(t)\}|$.

In a language $L$, a complete atom is a complete term $t$ such that $t([])$ is a predicate symbol.

Definition 2.3. The depth $\mathrm{dp}(t)$ of a term $t$ is defined by:

(i) $\operatorname{dp}(t)=\infty$, if $t$ is infinite

(ii) $\operatorname{dp}(t)=1+\max \{|n|: n \in \operatorname{dom}(t)\}$, if $t$ is finite.

The tree $\operatorname{dom}(t)$ is called the underlying tree of $t$. The set of all complete terms over $S$ is denoted by Term $_{S}$; these terms can be looked at as (possibly) infinite terms. By definition a term $t$ is finite if and only if $\operatorname{dom}(t)$ is finite. Next, we will define a metric on Term $_{S}$.

Definition 2.4. Let $s, t \in \operatorname{Term}_{S}$ and $s \neq t$ then we define $\beta(s, t)$ as being the least depth at which $s$ and $t$ differ. Then we define:

(i) $d(s, t)=0$ if $s=t$

(ii) $d(s, t)=2^{-\beta(s, t)}$ if $s \neq t$.

Proposition 2.5. $\left(\right.$ Term $\left._{S}, d\right)$ is an (ultra-)metric space.

The proof is simple, and omitted here. Note that the larger the depth is at which two terms differ, the smaller is their distance. Next, we define the truncation of a term, to have finite approximations of infinite terms. Assume $\Omega$ to be an extra constant symbol (hence with arity zero), not in $S$ (nor in Term $_{S}$ ).

Definition 2.6. The truncation at depth $n$ of a term $t$, notation $\alpha_{n}(t)$, can be found from the complete term $t$ by replacing all symbols at depth $n$ by $\Omega$ and leaving out all symbols at greater depth. The underlying tree $\operatorname{dom}\left(\alpha_{n}(t)\right)$ is adjusted in the same way, by leaving out all nodes without a label. 
Definition 2.7. A metric space $(X, d)$ is compact if every sequence in $X$ has a subsequence which converges to a point in $X$.

Proposition 2.8. $\left(\right.$ Term $\left._{S}, d\right)$ is compact iff $S$ is finite.

For a proof of this well known theorem, see [12]. From Definition 2.6 we find: $\operatorname{dp}\left(\alpha_{n}(t)\right) \leqslant n+1$, for all $t$. Moreover, $d\left(\alpha_{n}(t), t\right) \leqslant 2^{-n}$ and therefore $\lim _{n \rightarrow \infty} \alpha_{n}(t)=$ $t$. Next we will consider complete Herbrand models for a program $P$, having all possibly infinite terms as its universe.

Definition 2.9. Let $P$ be a program, then the complete Herbrand universe $\mathrm{CU}_{P}$ is defined by $\operatorname{Term}_{L(P)}$. A complete Herbrand model for $L_{P}$ is a model $\mathcal{M}$ with domain $\mathrm{CU}_{P}$, such that

(i) $a^{\cdot \text { "l }}=a$, for all constants $a \in L_{P}$

(ii) $f^{\cdot \mathcal{H}}\left(t_{1}, \ldots, t_{k}\right)=f\left(t_{1}^{\prime \prime}, \ldots, t_{k}^{\prime \mu}\right)$, for all functions $f \in L_{P}$ and complete terms $t_{1}, \ldots, t_{k} \in \operatorname{Term}_{L(P)}$.

A complete Herbrand model for a program $P$ is a complete Herbrand model for $L_{P}$ which satisfies $P$.

Definition 2.10. The complete Herbrand base $\mathrm{CB}_{P}$, or $\mathrm{CB}_{L(P)}$, of a program $P$ is defined by

$$
\mathrm{CB}_{P}=\left\{t \in \mathrm{CU}_{P}: t \text { is a complete atom }\right\} .
$$

The elements of $\mathrm{CB}_{P}$ can be represented as trees as well. Moreover, the metric $d$ on $\mathrm{CU}_{P}$ can be extended to $\mathrm{CB}_{P}$. Informally, we will write $U_{P}$ for the set of all finite terms in $L_{P}$ and $B_{P}$ for the set of all finite elements from $\mathrm{CB}_{P}$.

In general any complete Herbrand model for a program $P$, can be associated with a subset of the complete Herbrand base $\mathrm{CB}_{P}$ : such a subset then denotes the complete set of "ground" atoms, holding in the model. For any ground atom $\boldsymbol{A}$ and Herbrand model $\mathcal{M}$, we will use both notations $A \in \mathcal{M}$ and $\mathcal{M} \vDash A$, to express that $A$ holds in the model $\mathcal{M}$ and $\vDash A$ to express that $A$ holds in all (possibly nonHerbrand) models.

\section{Recursive specifications}

In this section we consider so called recursive specifications, which are finite sets of positive equational formulas and will be used later instead of the usual notion of a substitution. Returning to Prolog, we will need a different unification algorithm, since we will work in complete Herbrand models. 
Definition 3.1. Let $P$ be a program. Then the theory $\operatorname{Eq}\left(L_{P}\right)$ (or $\operatorname{Eq}(P)$ ) consists of the axioms:

(1) $c \neq d$ for all pairs of distinct constants $c, d$ from $L_{P}$

(2) $\forall x . f(x) \neq g(x)$ for all pairs of distinct function symbols $f, g$ from $L_{P}$

(3) $\forall \boldsymbol{x} . f(\boldsymbol{x}) \neq c$ for all function symbols $f$ and all constants $c$ from $L_{P}$

(4) $\forall x y . x_{1} \neq y_{1} \vee \cdots \vee x_{k} \neq y_{k} \rightarrow f(x) \neq f(\boldsymbol{y})$ for all function symbols $f$ from $L_{P}$

(5) $\forall x . x=x$

(6) $\forall x y . x_{1}=y_{1} \wedge \cdots \wedge x_{k}=y_{k} \rightarrow f(\boldsymbol{x})=f(\boldsymbol{y})$ for all function symbols $f$ from $L_{P}$

(7) $\forall x y . \quad x_{1}=y_{1} \wedge \cdots \wedge x_{k}=y_{k} \rightarrow(P(x) \rightarrow P(\boldsymbol{y}))$ for all predicate symbols $P$ from $L_{P}$.

Lemma 3.2. $\mathrm{Eq}(L)$ holds in all complete Herbrand models for $L$.

The axioms of $\operatorname{Eq}\left(L_{P}\right)$ are introduced in [12] to model finite failure: $\operatorname{Eq}\left(L_{P}\right)$ forces any two syntactically different terms to be different in all its models. In [12] we even find an extra axiom:

(8) $\forall x . x \neq t[x]$

for all terms $t$ that are unequal to a variable containing the variable $x$. This axiom is needed to express that the elements in the Herbrand universe consist of all finite terms from $L_{P}$. Since in the complete Herbrand universe we have infinite terms as well, we will omit axiom (8) from our equational theory. It turns out to be convenient to consider substitutions no longer as a syntactic operation of binding variables, but directly as equational formulas.

Definition 3.3. A (recursive) specification in a language $L$ is a set of equations of the form: $\left\{t_{1}(\boldsymbol{x})=s_{1}(\boldsymbol{x}), \ldots, t_{k}(\boldsymbol{x})=s_{k}(\boldsymbol{x})\right\}$ for (open) terms $t_{i}, s_{i} \in L$ and variables $\boldsymbol{x} \equiv x_{1}, \ldots, x_{n}$.

Definition 3.4. An open complete term in a language $L$ is obtained by constructing a complete term from $L \cup\left\{x_{i}: i \leqslant n\right\}$, where $\left\{x_{i}: i \leqslant n\right\}$ denotes a finite set of variable symbols with arity zero.

Note that an open complete term only has finitely many variables, called the free variables of the term. We will write $t(\boldsymbol{x})$ for the term $t$ which has variables only from $\boldsymbol{x} \equiv x_{1}, \ldots, x_{n}$ (but possibly less) and similarly we write $\rho(\boldsymbol{x})$ for a specification with variables only from $x \equiv x_{1}, \ldots, x_{n}$. Note that the metric $d$ can simply be generalised to open complete terms, by extending the language $L$ with extra variable symbols.

Proposition 3.5. Let $L$ be a language with at least one constant, and let $M$ be a complete Herbrand model for $L$. Then for all open complete terms $t_{1}(\boldsymbol{x}), t_{2}(\boldsymbol{x})$ we have:

$$
\left(\forall s \in U_{L}: \quad M \vDash t_{1}(s)=t_{2}(s)\right) \Leftrightarrow d\left(t_{1}(x), t_{2}(x)\right)=0 .
$$


Definition 3.6. A specification $\rho(x)$ is said to be in reduced form if it is of the form $\left\{x_{1}=s_{1}\left(x_{1}, \ldots, x_{n}\right), \ldots, x_{k}=s_{k}\left(x_{1}, \ldots, x_{n}\right)\right\}$, where $x_{1}, \ldots, x_{k}$ are distinct variables. Moreover, $\rho(\boldsymbol{x})$ has a reduced form it it is equivalent to a specification which is in reduced form. A specification is said to be in contradictory form if it contains an equation $a=b$ or $f\left(t_{1}, \ldots, t_{n}\right)=g\left(s_{1}, \ldots, s_{n}\right)$ for some distinct symbols $a, b$ or $f$, $g$, respectively. Moreover, it has a contradictory form if it is logically equivalent to a specification which is in contradictory form.

Definition 3.7. A variable $x$ is bound in $\rho$ if $\rho \models x=t$ for some term $t$ which is not a variable. Otherwise it is called free. A specification is called ground if it has no free variables.

Example. Let $\sigma(x, y)=\{x=f(x), y=x\}$, then $\sigma$ has no free variables since $\sigma \models x=$ $f(x)$ and $\sigma \models y=f(x)$. Let $\sigma(x, y, z)=\{x=f(y), y=z\}$, then $x$ is a bound variable in $\sigma$, whereas $y$ and $z$ are free.

Definition 3.8. A specification $\rho(x)$ is called consistent if $\rho \cup \operatorname{Eq}(\rho)$ is satisfiable in a model.

Theorem 3.9. Let $L$ be a language. If $\rho(x, y)$ is in reduced form, with bound variables $\boldsymbol{x} \equiv x_{1}, \ldots, x_{n}$ which are distinct and free variables $\boldsymbol{y}$, then there exist (open) complete terms $t_{1}(\boldsymbol{y}), \ldots, t_{n}(\boldsymbol{y})$ with only free variables from $\rho$, such that in every complete Herbrand model $M$ for $L$ :

$$
\mathcal{M} \vDash \forall x y . \rho(x, y) \leftrightarrow\left(x_{1}=t_{1}(y) \wedge \cdots \wedge x_{n}=t_{n}(y)\right) .
$$

Proof. Use the fact that for all equations $x=t(x, y)$ in $\rho, \mathcal{M} \vDash \forall x y . x=t(x, y) \leftrightarrow$ $x=z\left[z / t^{\omega}(y)\right]$, in every complete Herbrand model $\mathcal{M}$ for $L_{p}$ and with $t^{\omega}(y):=$ $t(t(t(\ldots, y), y), y)$.

Theorem 3.10. (i) A specification in reduced form is consistent.

(ii) A specification in contradictory form is inconsistent.

Proof. (i) Suppose $\rho$ is in reduced form, with language $L$. Extend $L$ until it contains at least one constant, then $L$ has a complete Herbrand model $M$. Then from Theorem 3.9 and Lemma 3.2 it follows that: $\mathcal{M} \vDash \rho \cup \operatorname{Eq}(\rho)$, thus $\rho$ is consistent. Part (ii) follows directly from the definition of $\operatorname{Eq}(\rho)$.

Corollary 3.11. Every specification in reduced form with a constant, has a complete Herbrand model.

Theorem 3.12. (i) All consistent specifications have a reduced form.

(ii) All inconsistent specifications have a contradictory form.

The proof of Corollary 3.11 follows from the proof of Theorem 3.10. Theorem 3.12 is the reverse of Theorem 3.10. In order to prove it, an algorithm can be 
constructed which actually decides whether a specification has a reduced form. This algorithm consists of the following five steps, defined in [2] (see also [8]). Suppose $\rho$ is a specification.

\section{Consistency algorithm}

(1) Delete from $\rho$ all equations of the form $x=x$.

(2) If $\rho$ contains an equation $x=y$, where $x$ and $y$ are different variables, then replace $x$ in all its occurences in $\rho$ by $y$.

(3) Replace an equation $t=x$ in $\rho$ by $x=t$, where $t$ is not a variable.

(4) Replace two equations $x=t$ and $x=s$ in $\rho$ by the equations $x=t$ and $t=s$, where $t$ is not larger than $s$ (in number of symbols).

(5) Replace an equation of the form $f\left(t_{1}, \ldots, t_{n}\right)=f\left(s_{1}, \ldots, s_{n}\right)$ by the equations $t_{1}=s_{1}, \ldots, t_{n}=s_{n}$.

It is well known that using these five steps repeatedly, any recursive specification $\rho$ can be reduced into a specification which is either in reduced form or in contradictory form, and equivalent to $\rho$. This provides us with a proof of Theorem 3.12. Furthermore, using the consistency algorithm one can define a new kind of unification which precisely coincides with unification in Prolog II. Assume $p$ is a predicate symbol and $S$ is a set of atoms.

\section{Unification algorithm}

(1) If not all atoms in $S$ start with the same predicate symbol, then $S$ is not unifiable.

(2) Else: if $S=\left\{p\left(t_{1}^{(i)}, \ldots, t_{n}^{(i)}\right): i \leqslant m\right\}$ then apply the consistency algorithm to $\left\{t_{1}^{(1)}=t_{1}^{(2)}, t_{1}^{(2)}=t_{1}^{(3)}, \ldots, t_{1}^{(m-1)}=t_{1}^{(m)}, \ldots, t_{n}^{(1)}=t_{n}^{(2)}, t_{n}^{(2)}=t_{n}^{(3)}, \ldots, t_{n}^{(m-1)}=t_{n}^{(m)}\right\}$.

Corollary 3.13. A specification $\rho$ is consistent iff it has a reduced form.

Definition 3.14. Let $S$ be a set of (open) atoms. A specification $\rho$ is called complete unifier (cu) for $S$, if $\models \forall\left(\rho \rightarrow \bigwedge_{A, B \in S}(A \leftrightarrow B)\right)$. Suppose $\rho$ is a cu for $S$. $\rho$ is called most general complete unifier (mcu) for $S$, if for all complete unifiers $\rho_{1}$ for $S$ and all complete Herbrand models $\mathcal{M}, \mathcal{M} \models \forall\left(\rho_{1} \rightarrow \rho\right)$.

Proposition 3.15. For any input set $S$ of atoms, the unification algorithm computes an mcu for $S$.

Note that Proposition 3.15 does not hold if we change Definition 3.14 by requiring $\forall\left(\rho_{1} \rightarrow \rho\right)$ to hold in all models instead of only complete Herbrand models. For instance if $s=\{p(x), p(f(f(x))\}$ then $\{x=f(f(x))\}$ is computed by the unification algorithm, although $\{x=f(x)\}$ is more general. With respect to complete Herbrand models, however, they are equivalent.

Theorem 3.16. Let $\rho$ be a (possibly infinite) specification in a language $L$ with at least one constant, then the following are equivalent:

(i) $\rho \cup \operatorname{Eq}(\rho)$ has a model, 
(ii) $\rho$ has a complete Herbrand model,

(iii) $\rho$ is satisfiable in all complete Herbrand models for $L$.

Proof. (ii) $\Rightarrow$ (iii) and (iii) $\Rightarrow$ (i) are easy and left to the reader.

(i) $\Rightarrow$ (ii): suppose $\rho=\cup_{i \in \omega} \rho_{i}\left(x_{i}\right)$, where all $\rho_{i}\left(x_{i}\right)$ are finite subsets of $\rho$, and define for all $n: \sum_{n}\left(x_{1}, \ldots, x_{n}\right)=\bigcup_{i \leqslant n} \rho_{i}\left(x_{i}\right)$. Clearly $\sum_{n}$ is finite and consistent and hence equivalent to a reduced form $\sum_{n}^{\prime}$. From Theorem 3.9 it follows that $\mathcal{M} \vDash \sum_{n}^{\prime}\left(t_{1}^{n}, \ldots, t_{n}^{n}\right)$ for all complete Herbrand models $\mathcal{M}$. Thus, for every natural number $n$ we obtain a sequence $\left(\boldsymbol{t}_{n}^{i}\right)_{i \geqslant n}$ in $\mathrm{CU}_{\rho}$. Since $\mathrm{CU}_{\rho}$ is compact every such sequence has a subsequence $\left(\boldsymbol{t}_{n}^{i}\right)_{i \geqslant n}$ which converges to a limit $\boldsymbol{t}_{n}$. By topological arguments we find $\mathcal{M} \vDash \sum_{n}^{\prime}\left(t_{1}, \ldots, t_{n}\right)$ for all $i \geqslant n$, so $\mathcal{M} \vDash \sum_{n}\left(t_{1}, \ldots, t_{n}\right)$ for all $n$, i.e. $\rho=\bigcup_{n \in \omega} \sum_{n}$ is satisfiable in all $M$.

Theorem 3.17. Let $L$ be a language, and suppose $A(x), B(x) \in L$ are unifiable atoms with $\mathrm{mcu} \rho(\boldsymbol{x})$ then for all complete Herbrand models $\mathcal{M}$ for $L$ and all $\boldsymbol{t} \equiv t_{1}, \ldots, t_{k} \in$ $|\mathcal{M}|$, the following are equivalent:

(i) $M \vDash \rho(t)$,

(ii) $d(A(t), B(t))=0$.

Proof. (i) $\Rightarrow$ (ii): if $\mathcal{M} \vDash \rho(t)$ for some complete Herbrand model $\mathcal{M}$, then it follows easily from Theorem 3.16 and Definition 3.14 that for all $\mathcal{M}$, we have $\mathcal{M} \vDash A(t) \leftrightarrow$ $B(\boldsymbol{t})$. Hence $d(A(\boldsymbol{t}), B(\boldsymbol{t}))=0$.

(ii) $\Rightarrow$ (i): $\quad$ if $\quad d(A(t), \quad B(\boldsymbol{t}))=0 \quad$ where: $\quad A=p\left(u_{1}(\boldsymbol{x}), \ldots, u_{n}(\boldsymbol{x})\right), \quad B=$ $p\left(v_{1}(\boldsymbol{x}), \ldots, v_{n}(\boldsymbol{x})\right)$ then $d\left(u_{i}(\boldsymbol{t}), \quad v_{i}(\boldsymbol{t})\right)=0$ for all $i$, and hence $\rho^{\prime}=$ $\left\{u_{1}(\boldsymbol{x})=v_{1}(\boldsymbol{x}), \ldots, u_{n}(\boldsymbol{x})=v_{n}(\boldsymbol{x})\right\}$ is consistent. Since $\rho^{\prime}$ is a cu for $\{A, B\}$, and $\rho$ is an mcu, it follows by Definition 3.14 that $M \vDash \rho(t)$.

\section{Complete SLD-resolution}

In this section we will assume $P$ to be some fixed program, with at least one constant symbol in its language $L_{P}$. Specifications will be denoted by $\sigma, \rho, \tau, \ldots$ For convenience, we present a few notations in the following definition.

Definition 4.1. For specifications $\sigma(\boldsymbol{x}), \rho(\boldsymbol{x})$ with variables $\boldsymbol{x} \equiv x_{1}, \ldots, x_{k}$ and for arbitrary complete terms $\boldsymbol{u} \equiv u_{1}, \ldots, u_{k}$ we write:

$$
\begin{aligned}
& u \in \sigma: \Leftrightarrow \text { for all complete Herbrand models } \mathcal{M} \text { for } L_{P}: \mathcal{M} \vDash \sigma[\boldsymbol{u}], \\
& \sigma \equiv \rho: \Leftrightarrow \models \forall(\sigma \leftrightarrow \rho), \\
& \sigma \leqslant \rho: \Leftrightarrow \models \forall(\sigma \rightarrow \rho) \quad(\text { ' } \sigma \text { is more specific than } \rho \text { '), } \\
& \sigma \equiv \perp: \Leftrightarrow \sigma \text { is inconsistent ( } \perp \text { stands for the bottom in the ordering } \leqslant), \\
& \rho \equiv \operatorname{mcu}(\{A, B\}): \Leftrightarrow \rho \text { is an mcu for } A \text { and } B .
\end{aligned}
$$

Furthermore we often write $\sigma \cdot \rho$ or $\sigma \rho$ instead of $\sigma \cup \rho$. 
Proposition 4.2. One can easily check the following statements:

(i) $(\emptyset \cdot \sigma) \equiv \sigma,(\perp \cdot \sigma) \equiv \perp,(\sigma \cdot \rho) \equiv(\rho \cdot \sigma),((\sigma \cdot \rho) \cdot \tau) \equiv(\sigma \cdot(\rho \cdot \tau))$.

(ii) $\sigma \leqslant \rho \Leftrightarrow$ for some $\tau$ : $\sigma \equiv(\rho \cdot \tau)$.

(iii) if $\rho(\boldsymbol{x})$ is ground (i.e. has no free variables) and $\sigma(\boldsymbol{x}, \boldsymbol{y}), \rho(\boldsymbol{x}, \boldsymbol{z})$ are specifications with distinct variables $\boldsymbol{x}, \boldsymbol{y}$ and $\boldsymbol{z}$, then

$$
\rho \sigma \tau(x, y, z) \equiv \perp \Leftrightarrow \rho \sigma(x, y) \equiv \perp \vee \rho \tau(x, z) \equiv \perp .
$$

The proof of Proposition 4.2 is easy and left to the reader. Taking $\rho=\emptyset$, it follows from (iii) that $\sigma(y) \cdot \tau(z) \equiv \perp$ if and only if $\sigma(y) \equiv \perp$ or $\tau(z) \equiv \perp$.

Next, we will make straightforward adaptations to some well-known definitions.

Definition 4.3. A goal is a pair $(G, \sigma)$ where $G$ is a goal clause and $\sigma$ is a specification.

Definition 4.4. A computation rule (c-rule, for short) is a function $R$ from goals $\left(\leftarrow A_{1}, \ldots, A_{k}, \sigma\right)$ to atoms $A$, such that $A \in\left\{A_{1}, \ldots, A_{k}\right\}$.

Definition 4.5. Let $G_{i}$ be the goal $\left(\leftarrow A_{1}, \ldots, A_{m}, \ldots, A_{k}, \sigma\right)$ and $C_{i+1}=A \leftarrow$ $B_{1}, \ldots, B_{q}$ a clause and $R$ a c-rule. Now, $G_{i+1}$ is derived from $G_{i}$ and $C_{i+1}$ by $R$ and $\rho$, if

(i) $R\left(G_{i}\right)=A_{m}$ and $G_{i+1}=\left(\leftarrow A_{1}, \ldots, A_{m-1}, B_{1}, \ldots, B_{q}, A_{m+1}, \ldots, A_{k}, \sigma \rho\right)$

(ii) $\sigma \neq \equiv \perp$ and $\rho \equiv \operatorname{mcu}\left(\left\{A_{m}, A\right\}\right)$.

Definition 4.6. Let $G$ be a goal and $R$ a c-rule. A CSLD-derivation for $P \cup\left\{G_{0}, \sigma_{0}\right\}$ is a sequence $\left(G_{0}, \sigma_{0}\right), \ldots,\left(G_{k}, \sigma_{k}\right), \ldots$, such that for some sequence of program clauses $C_{1}, \ldots, C_{k}, \ldots \in P$ with new variables, $G_{i+1}$ is derived from $G_{i}$ and $C_{i+1}$ by $R$, for all $i$. Note that if $\sigma_{k} \equiv \perp$ then $\left(G_{k}, \sigma_{k}\right)$ is the last goal in the derivation. A CSLD-refutation is a CSLD-derivation with $(\square, \rho)$ as the last goal, where $\square$ stands for the empty goal clause, and $\rho$ is a consistent specification which is called the computed answer specification for $P \cup\left\{G_{0}, \sigma_{0}\right\}$ with c-rule $R$.

Definition 4.7. A correct answer specification for $P \cup\left(\leftarrow A_{1}, \ldots, A_{k}, \rho\right)$ is a consistent specification $\sigma$ such that $P \models \forall\left(\sigma \rightarrow A_{1} \wedge \cdots \wedge A_{k} \wedge \rho\right)$.

CSLD-derivation stands for complete SLD-derivation. Note that such derivations are logical derivations as well, as is stated in the next soundness theorem, originally due to Van Emden and Lloyd [6].

Theorem 4.8 (soundness of CSLD-resolution). Computed answer specifications are correct.

Proof. Let $\left(G_{i}, \sigma_{i}\right)_{0 \leqslant i \leqslant n}$ be a CSLD-refutation for $P \cup\left(\leftarrow A_{1}, \ldots, A_{k}, \sigma_{0}\right)$. By induction we prove (induction hypothesis) $P \vDash \forall\left(\sigma_{n} \rightarrow A_{1} \wedge \cdots \wedge A_{k} \wedge \sigma_{0}\right)$. 
( $n=1)$ : Now $G_{0}$ is of the form $\left(\leftarrow A_{1}\right)$ and $P$ has a unit clause $A \leftarrow$ such that $\sigma_{0} \rho$ is consistent, where $\rho \equiv \operatorname{mcu}\left(\left\{A_{1}, A\right\}\right)$. By Definition 3.14 we have $\models \forall\left(\rho \rightarrow\left(A_{1} \leftrightarrow A\right)\right)$ and since $\sigma_{1} \equiv \sigma_{0} \rho \leqslant \rho$ (see Proposition 4.1) we find $\models \forall\left(\sigma_{1} \rightarrow\left(A_{1} \leftrightarrow A\right)\right.$ ). Since $(A \leftarrow) \in P$, we have $P \vDash \forall(A)$ and therefore $P \vDash \forall\left(\sigma_{1} \rightarrow A_{1}\right)$, hence $P \models \forall\left(\sigma_{1} \rightarrow A_{1} \wedge\right.$ $\left.\sigma_{0}\right)$, since $\sigma_{1} \leqslant \sigma_{0}$.

$(n+1)$ : Assume $R\left(G_{0}\right)=A_{i}$, then there is a clause $A \leftarrow B_{1}, \ldots, B_{q} \in P$ such that $\sigma_{1} \equiv \sigma_{0} \rho$ is consistent, where $\rho \equiv \operatorname{mcu}\left(\left\{A_{i}, A\right\}\right)$ (see Definitions 4.5 and 4.6). Now $\left(G_{i}, \sigma_{i}\right)_{1 \leqslant i \leqslant n+1}$ is a CSLD-refutation for

$$
P \cup\left\{\left(\leftarrow A_{1}, \ldots, A_{i-1}, B_{1}, \ldots, B_{q}, A_{i+1}, \ldots, A_{k}, \sigma_{1}\right)\right\}
$$

with length $n$, so by induction we obtain:

(i) $P \vDash \forall\left(\sigma_{n+1} \rightarrow A_{1} \wedge \cdots \wedge A_{i-1} \wedge B_{1} \wedge \cdots \wedge B_{q} \wedge A_{i+1} \wedge \cdots \wedge A_{k} \wedge \sigma_{1}\right)$

(ii) $P \models \forall\left(\sigma_{n+1} \rightarrow A_{1} \wedge \cdots \wedge A_{i-1} \wedge A_{i+1} \wedge \cdots \wedge A_{k} \wedge \sigma_{0}\right)$.

Here (ii) follows from $\sigma_{1} \leqslant \sigma_{0}$. By Definition 3.14 it follows that $\models \forall\left(\rho \rightarrow\left(A_{i} \leftrightarrow A\right)\right)$, and since $A \leftarrow B_{1} \wedge \cdots \wedge B_{q} \in P$ we have $P \models \forall\left(B_{1} \wedge \cdots \wedge B_{q} \wedge \rho \rightarrow A_{i}\right)$. Therefore from $\sigma_{n+1} \leqslant \sigma_{0} \rho \leqslant \rho$ and (i) we find that $P \models \forall\left(\sigma_{n+1} \rightarrow A_{i}\right)$. Hence, with (ii), $P \models \forall\left(\sigma_{n+1} \rightarrow A_{1} \wedge \cdots \wedge A_{k} \wedge \sigma_{0}\right)$.

Next we will consider a method, first introduced in [5], to find models for logic programs using fixed points of some monotonic mapping. We assume $A(\boldsymbol{x})$ to be finite for variables $x$.

Definition 4.9. For $X \subseteq \mathrm{CB}_{P}$ we define

$$
\begin{aligned}
\mathrm{CT}_{P}(X):= & \left\{\boldsymbol{A}(\boldsymbol{u}) \in \mathrm{CB}_{P}: \text { there are terms } \boldsymbol{v} \in \mathrm{CU}_{P} \text { and a clause } A_{1}(\boldsymbol{x})\right. \\
& \leftarrow B_{1}(\boldsymbol{x}), \ldots, B_{q}(\boldsymbol{x}) \in P \text { such that } d\left(A(\boldsymbol{u}), A_{1}(\boldsymbol{v})\right)=0 \\
& \text { and } \left.\left\{B_{1}(\boldsymbol{v}), \ldots, B_{q}(\boldsymbol{v})\right\} \subseteq X\right\} .
\end{aligned}
$$

$\mathrm{CT}_{P}$ is a continuous mapping on the complete lattice formed by all subsets of $\mathrm{CB}_{P}$, with the usual ordering $\subseteq . \mathrm{CT}_{P}$ is often called the one step derivation map.

Definition 4.10. We will use the following notation:

$$
\begin{aligned}
& \mathrm{CT}_{P} \uparrow 0=\emptyset, \\
& \mathrm{CT}_{P} \uparrow k+1=\mathrm{CT}_{P}\left(\mathrm{CT}_{P} \uparrow k\right), \quad k \in \omega, \\
& \mathrm{CT}_{P} \uparrow \omega=\bigcup_{k<\omega} \mathrm{CT}_{P} \uparrow k .
\end{aligned}
$$

A well-known theorem says that $\mathrm{CT}_{P} \uparrow \omega$ is the least fixed point of $\mathrm{CT}_{P}$, denoted by If $\left(\mathrm{CT}_{P}\right)$. We write $T_{P}$ for the mapping defined on the powerset of $B_{P}$ which is $\mathrm{CT}_{P}$ but then restricted to finite atoms. 
Proposition 4.11. Let $P$ be a program and $M \subseteq \mathrm{CB}_{P}$ a complete Herbrand model, then $\mathcal{M}$ is a model for $P \Leftrightarrow \mathrm{CT}_{P}(\mathcal{M}) \subseteq \mathcal{M}$.

Proposition 4.12. Let $P$ be a program, then $\operatorname{lfp}\left(\mathrm{CT}_{P}\right)=\cap\left\{\mathscr{M} \subseteq \mathrm{CB}_{P}: \mathrm{CT}_{P}(\mathscr{M}) \subseteq \mathcal{M}\right\}$.

Corollary 4.13. (i) $\mathrm{CT}_{P} \uparrow \omega$ is a model for $P$.

(ii) $\mathrm{CT}_{P} \uparrow \omega=\cap\left\{M \subseteq \mathrm{CB}_{P}: \mathcal{M}\right.$ is a model for $\left.P\right\}$.

The proof of Proposition 4.11 is easy and left to the reader. Proposition 4.12 is a special instance of a general theorem in the theory of complete partial orders. Its proof is omitted here (see [12]). It follows from Corollary 4.13 that $\mathrm{CT}_{P} \uparrow \omega$ is the least complete Herbrand model for $P$.

Let us return to the notion of CSLD-resolution. We will see that the set of complete atoms with a CSLD-refutation coincides with the least fixed point $\mathrm{CT}_{P} \uparrow \omega$. First we present an important lemma.

Lemma 4.14 (mcu-lemma). Let $G$ be a goal and assume $P \cup\left\{G_{0}, \sigma_{0}\right\}$ has a CSLDrefutation $\left(G_{i}, \sigma_{i}\right)_{0 \leqslant i \leqslant n}$ with $\sigma_{i} \equiv \sigma_{0} \theta_{1} \cdots \theta_{i}$, where $\theta_{1}, \ldots, \theta_{i}$ are complete unifiers, but not necessarily most general complete. Then there is a CSLD-refutation $\left(G_{1}^{\prime}, \sigma_{i}^{\prime}\right)_{0 \leqslant i \leqslant n}$ of the same length, such that:

(i) $\sigma_{1}^{\prime} \equiv \sigma_{0} \theta_{1}^{\prime} \cdots \theta_{i}^{\prime}$

(ii) $\theta_{1}^{\prime}, \ldots, \theta_{i}^{\prime}$ are most general complete unifiers

(iii) $\sigma_{n} \leqslant \sigma_{n}^{\prime}$.

The proof is an analogon of the proof given in [12]. In fact the mcu-lemma states that refutations with only complete unifiers, can be turned into a more general refutation by using most general complete unifiers. Let us define the complete equivalent of the success set (see [1]).

Definition 4.15. The complete success set $\mathrm{CS}_{P}$ of a program $P$ is defined by

$$
\begin{aligned}
\mathrm{CS}_{P}=\{ & A(\boldsymbol{u}) \in \mathrm{CB}_{P}: P \cup\{(\leftarrow A(\boldsymbol{x}), \emptyset)\} \text { has a CSLD-refutation with } \\
& \text { computed answer specification } \sigma(\boldsymbol{x}, \boldsymbol{y}), \text { such that for some } \\
& \boldsymbol{v}:(\boldsymbol{u}, \boldsymbol{v}) \in \sigma\} .
\end{aligned}
$$

Proposition 4.16. $\mathrm{CS}_{P}$ is well defined.

Proof. Assume $d\left(A_{1}(\boldsymbol{u}), A_{2}(\boldsymbol{w})\right)=0$ and $A_{1}(\boldsymbol{u}) \in \mathrm{CS}_{P}$, then we prove that $A_{2}(\boldsymbol{w}) \in$ $\mathrm{CS}_{P}$. By definition $\left(\leftarrow A_{1}(x), \emptyset\right)$ has a CSLD-refutation $\left(G_{i}, \sigma_{i}\right)_{0 \leqslant i \leqslant n}$ with computed answer specification $\sigma_{n}(\boldsymbol{x}, \boldsymbol{y})$ such that for some $\boldsymbol{v}:(\boldsymbol{u}, \boldsymbol{v}) \in \sigma_{n}$. Let $\rho(\boldsymbol{x}, \boldsymbol{z}) \equiv$ $\operatorname{mcu}\left(\left\{\boldsymbol{A}_{1}(\boldsymbol{x}), \boldsymbol{A}_{2}(\boldsymbol{z})\right\}\right)$, then $\rho$ is consistent and $(\boldsymbol{u}, \boldsymbol{w}) \in \rho$. Clearly $(\boldsymbol{u}, \boldsymbol{v}, \boldsymbol{w}) \in \rho_{n}(\boldsymbol{x}, \boldsymbol{y}, \boldsymbol{z})$ and therefore $\rho \sigma_{n}$ is consistent and $\left(G_{i}, \rho \sigma_{i}\right)_{0 \leqslant i \leqslant n}$ is a refutation for $P \cup\left\{\leftarrow A_{2}(z), \rho\right\}$ (using the same clauses and the same computation rule). Now consider $\left(\leftarrow A_{2}(z), \emptyset\right)$, $\left(G_{1}, \rho \sigma_{1}\right),\left(G_{2}, \rho \sigma_{2}\right), \ldots,\left(G_{n}, \rho \sigma_{n}\right)$ then clearly this is a refutation for $P \cup$ $\left\{\leftarrow A_{2}(z), \emptyset\right\}$ except that $\rho \sigma_{1}$ is not most general. Thus by Lemma 4.14 there is a 
refutation for $P \cup\left\{\leftarrow A_{2}(z), \emptyset\right\}$ with computed answer specification $\sigma(x, y, z) \geqslant$ $\rho \sigma_{n}(\boldsymbol{x}, \boldsymbol{y}, \boldsymbol{z})$, so $(\boldsymbol{u}, \boldsymbol{v}, \boldsymbol{w}) \in \sigma$. Hence $A_{2}(\boldsymbol{w}) \in \mathrm{CS}_{P}$.

Theorem 4.17. $\mathrm{CS}_{P}=\mathrm{CT}_{P} \uparrow \omega$.

Proof. $(\Rightarrow)$ : Let $A(\boldsymbol{u}) \in \mathrm{CS}_{P}$ then $P \cup\{(\leftarrow A(\boldsymbol{x}), \emptyset)\}$ has a CSLD-refutation with answer specification $\sigma(x, y)$, such that for some $v:(\boldsymbol{u}, \boldsymbol{v}) \in \sigma$. By soundness of CSLD-resolution it follows that $\sigma(x, y)$ is correct for $P \cup\{(\leftarrow A(x)$, $\emptyset)\}$, i.e. $P \models \forall(\sigma(x, y) \rightarrow A(x))$, and hence $M \vDash A(u)$ for all complete Herbrand models $M$ for $P$, since $(\boldsymbol{u}, \boldsymbol{v}) \in \sigma$. Then by Corollary 4.13 it follows that $A(\boldsymbol{u}) \in \mathrm{CT}_{P} \uparrow \omega$.

$(\Leftarrow)$ : Let $A(\boldsymbol{u}) \in \mathrm{CT}_{P} \uparrow \omega$ then $A(\boldsymbol{u}) \in \mathrm{CT}_{P} \uparrow n$, for some $n<\omega$. Now the proof proceeds by induction.

$(\boldsymbol{n}=1)$ : Then $A(\boldsymbol{u}) \in \mathrm{CT}_{P}(\emptyset)$, so there is a unit clause $A_{1}(\boldsymbol{y}) \leftarrow \in P$, such that for some $\boldsymbol{v},(\boldsymbol{u}, \boldsymbol{v}) \in \rho(\boldsymbol{x}, \boldsymbol{y})$ and $\rho(\boldsymbol{x}, \boldsymbol{y}) \equiv \operatorname{mcu}\left(\left\{\boldsymbol{A}(\boldsymbol{x}), A_{1}(\boldsymbol{y})\right\}\right)$; since $\rho$ is consistent, $(\leftarrow A(x), \emptyset),(\square, \rho(x, y))$ is a CSLD-refutation for $P \cup\{\leftarrow A(x), \emptyset\}$ with answer specification $\rho(\boldsymbol{x}, \boldsymbol{y})$.

$(\boldsymbol{n}+1)$ : Now, $A(\boldsymbol{u}) \in \mathrm{CT}_{P}\left(\mathrm{CT}_{P} \uparrow n\right)$, so there is a clause $A_{1}(\boldsymbol{y}) \leftarrow B_{1}(y), \ldots, B_{q}(y) \in$ $P$ and $\boldsymbol{v}$ such that: $A(\boldsymbol{u}) \equiv A_{1}(\boldsymbol{v})$ and $B_{1}(\boldsymbol{v}), \ldots, B_{q}(\boldsymbol{v}) \in \mathrm{CT}_{P} \uparrow n$. Assume $\rho(\boldsymbol{x}, \boldsymbol{y}) \equiv$ $\operatorname{mcu}\left(\left\{A(\boldsymbol{x}), A_{1}(\boldsymbol{y})\right\}\right)$ then $(\boldsymbol{u}, \boldsymbol{v}) \in \rho(\boldsymbol{x}, \boldsymbol{y})$. By the induction hypothesis, there exist refutations for $P \cup\left\{\leftarrow B_{i}(\boldsymbol{y}), \emptyset\right\}$ with computed answer specification $\sigma_{i}\left(\boldsymbol{y}, \boldsymbol{z}_{i}\right)$ such that for some $\boldsymbol{w}_{i},\left(\boldsymbol{v}, \boldsymbol{w}_{i}\right) \in \sigma_{i}$. These refutations can be put together to obtain a new refutation for $P \cup\left\{\leftarrow B_{1}(y), \ldots, B_{q}(y), \emptyset\right\}$ with a computed answer specification $\sigma \geqslant \sigma_{1} \cdots \sigma_{q}$. Since $\left(\boldsymbol{v}, \boldsymbol{w}_{1}, \ldots, \boldsymbol{w}_{q}\right) \in \sigma_{1} \cdots \sigma_{q}$, such a consistent $\sigma$ exists, and we directly find that $\sigma \rho$ is consistent as well, since $\left(\boldsymbol{u}, \boldsymbol{v}, \boldsymbol{w}_{1}, \ldots, \boldsymbol{w}_{q}\right) \in \sigma \rho$. Hence $\sigma \rho$ is the intended answer specification.

Theorem 4.17 is part of a more general result from [10] on a language with equations and inequations.

So far, we found that the correctness theorem can be restored for CSLD-resolution. Moreover, $\mathrm{CT}_{P} \uparrow \omega$ is the least complete Herbrand model which is the intersection of all complete Herbrand models for $P$, and equal to the complete success set. Next we will show that we have a completeness theorem as well.

Definition 4.18 (restriction). Let $\sigma(x, y)$ and $\rho(x, z)$ be two specifications then we write $\sigma \leqslant_{x} \rho$ if $\vDash \forall x y .(\sigma(x, y) \rightarrow \exists z . \rho(x, z))$. Furthermore we write $\sigma \equiv_{x} \rho$ if both $\sigma \leqslant_{x} \rho$ and $\rho \leqslant_{x} \sigma$.

Proposition 4.19. Let $\sigma(\boldsymbol{x})$ be ground then for all specifications $\rho$ : either $\sigma \rho \equiv \perp$ or $\sigma \leqslant_{x} \rho$.

Definition 4.18 is needed to indicate that $\sigma$ is more specific than $\rho$, although $\rho$ may bind variables not occurring in $\sigma$. Moreover, $\sigma \equiv_{x} \rho$ indicates that $\sigma$ and $\rho$ are 
equivalent with respect to the variables $x$. Note that $\sigma \equiv_{x} \perp \Rightarrow \sigma \equiv \perp$ for all variables $x$. Proposition 4.19 says that ground specifications cannot be further specified with respect to their variables: either $\sigma \cup \rho$ is inconsistent, or $\sigma$ is more specific than $\rho$ with respect to $x$. For example, let $\sigma(x)=\{x=f(x, x)\}$ and $\rho(x, y)=\{x=f(x, y)$, $y=x\}$ then $\sigma \leqslant_{x} \rho$, however not $\sigma \leqslant \rho$ (i.e. $\sigma \leqslant_{x y} \rho$ ), since $\rho$ has an extra variable $y$. In fact: $\sigma \equiv_{x} \rho$.

Lemma 4.20. Let $A(\boldsymbol{x})$ be an atom and $\sigma(\boldsymbol{x})$ correct for $P \cup\{\leftarrow A(\boldsymbol{x}), \emptyset\}$, then there exists a CSLD-refutation for $P \cup\{\leftarrow A(\boldsymbol{x}), \sigma(\boldsymbol{x})\}$ with $\rho(\boldsymbol{x}, \boldsymbol{y})$ as computed answer specification, such that $\sigma \equiv_{x} \rho$.

Proof. First, assume $\sigma(\boldsymbol{x})$ is ground. Now, let $\boldsymbol{u} \in \sigma$ then $A(\boldsymbol{u}) \in \mathrm{CT}_{P} \uparrow \omega$ (by Theorem 3.16, Definition 4.7 and Corollary 4.13). Therefore $\boldsymbol{A}(\boldsymbol{u}) \in \mathrm{CS}_{P}$ (by Theorem 4.17), hence there is a CSLD-refutation $\left(G_{i}, \rho_{i}\right)_{0 \leqslant i \leqslant n}$ for $P \cup\{\leftarrow A(x), \emptyset\}$ with computed answer specification $\rho_{n}(\boldsymbol{x}, \boldsymbol{y})$ such that for some $\boldsymbol{v},(\boldsymbol{u}, \boldsymbol{v}) \in \rho_{n}$. Because $(\boldsymbol{u}, \boldsymbol{v}) \in \sigma \rho_{n}$, $\sigma \rho_{n}$ is consistent and therefore $\left(G_{i}, \sigma \rho_{i}\right)_{0 \leqslant i \leqslant n}$ is a refutation for $P \cup\{\leftarrow A(\boldsymbol{x}), \sigma\}$ with computed answer specification $\sigma \rho_{n}$, and by Proposition 4.2 we have $\sigma \rho_{n} \leqslant \sigma$. Since $\sigma$ is ground we find by Proposition 4.19, $\sigma \leqslant_{x} \sigma \rho_{n}$. Hence $\sigma \equiv_{x} \sigma \rho_{n}$.

Next, assume $\sigma$ is not ground, and let $\boldsymbol{x}=(\boldsymbol{y}, \boldsymbol{z})$ where $\boldsymbol{y}$ and $\boldsymbol{z} \equiv z_{1}, \ldots, z_{k}$ are the bound and free variables of $\sigma$, respectively. Let $a \equiv a_{1}, \ldots, a_{k}$ be new constants not occurring in $P, A$ or $\sigma$, and such that for $i, j, a_{i}=a_{j} \Leftrightarrow \sigma \models z_{i}=z_{j}$. Next, consider $\sigma^{\prime}(\boldsymbol{y}, \boldsymbol{z}) \equiv\left\{z_{1}=a_{1}, \ldots, z_{n}=a_{n}\right\} \cdot \sigma(\boldsymbol{y}, \boldsymbol{z})$, then $\sigma^{\prime}$ is consistent and ground. Hence there exists a CSLD-refutation for $P \cup\left\{\leftarrow A, \sigma^{\prime}(y, z)\right\}$ with computed answer specification $\rho^{\prime}\left(y, z, z^{\prime}\right)$ such that $\sigma^{\prime} \equiv_{y z} \rho^{\prime}$, or equivalently: $\sigma^{\prime} \equiv_{x} \rho^{\prime}$. Now it is easy to see, that we can find a new refutation for $P \cup\{\leftarrow A, \sigma\}$ by replacing all constants $a$ by new variables, with computed answer specification $\rho$, such that $\sigma \equiv_{x} \rho$.

Note that Lemma 4.20 does not hold if we replace $\equiv_{x}$ by $\equiv$. For example let $P=\{A(y, y) \leftarrow\}$ and consider the goal clause $G=\leftarrow A(x, f(x))$ then one can easily see that $\sigma(x)=\{x=f(x)\}$ is correct for $P \cup\{G, \emptyset\}$. Indeed, there is a computed answer specification $\rho(x, y)=\{x=\{y, f(y)\}\}$ which is equivalent to $\rho^{\prime}(x, y)=\{x=y$, $y=f(y)\}$. Clearly $\rho \leqslant \sigma$, however since $\not \neq \forall(x=f(x) \rightarrow(x=y \wedge y=f(y)))$, the converse is not true. Hence $\rho \not \equiv \sigma$.

The point is, that in a CSLD-refutation new variables are introduced (input clauses have new variables), and the correct specification we started with cannot impose any constraints upon variables other than its own. In "common" SLD-resolution, this problem does not occur since computed substitutions are restricted to the goal variables automatically. This can be done, because in SLD-resolution new variables are bound to finite terms (not containing the variable again) hence one can simply carry out the substitution. This problem is overcome, however, by introducing $\leqslant_{x}$ as a logical notion of restriction. 
Lemma 4.21 (lifting lemma). Let $G$ be a goal clause and $\sigma$ be a specification. Assume there exists a CSLD-refutation for $P \cup\{G, \sigma\}$ with computed answer specification $\rho$. If $\sigma \leqslant \sigma^{\prime}$ then there is a CSLD-refutation for $P \cup\left\{G, \sigma^{\prime}\right\}$ with computed answer specification $\rho^{\prime}$ of the same length such that $\rho \leqslant \rho^{\prime}$.

Theorem 4.22 (completeness of CSLD-resolution). Let $\left(G_{0}(x), \sigma_{0}(x)\right)$ be a goal and $\sigma(x)$ a correct answer specification for $P \cup\left\{G_{0}(x), \sigma_{0}(x)\right\}$ then there exists a computation rule $R$ and a $R$-computed answer specification $\rho(x, y)$ for $P \cup\left\{G_{0}(x), \sigma_{0}(x)\right\}$ such that $\sigma \leqslant_{x} \rho$.

Proof. Assume $G_{0}=\left(\leftarrow A_{1}(\boldsymbol{x}), \ldots, A_{k}(\boldsymbol{x})\right)$ then $P \vDash \forall\left(\sigma \rightarrow A_{1}(\boldsymbol{x}) \wedge \cdots \wedge A_{k}(\boldsymbol{x}) \wedge \sigma_{0}\right)$ since $\sigma$ is correct for $P \cup\left\{G_{0}, \sigma_{0}\right\}$. By Lemma 4.18 there exist refutations for $P \cup\left\{\leftarrow A_{i}, \sigma\right\}$ with $\rho_{i}$ as computed answer specification, such that $\rho_{i} \equiv_{x} \sigma$ for all $i$. These refutations can be combined to obtain a new refutation for $P \cup$ $\left\{\leftarrow A_{1}(\boldsymbol{x}), \ldots, A_{k}(\boldsymbol{x}), \sigma\right\}$ with answer specification $\rho^{\prime} \equiv \rho_{1} \cdots \rho_{k}$, so $\sigma \equiv_{x} \rho^{\prime}$. Since $\sigma \leqslant \sigma_{0}$, it follows by the lifting lemma that there exists a CSLD-refutation for $P \cup\left\{\leftarrow A_{1}(\boldsymbol{x}), \ldots, A_{k}(\boldsymbol{x}), \sigma_{0}\right\}$ with computed answer specification $\rho$ such that $\rho^{\prime} \leqslant \rho$. Since $\sigma \equiv_{x} \rho^{\prime}$ we have $\sigma \leqslant_{x} \rho$.

The proof of Lemma 4.21 is similar to the one in [12]. The completeness theorem presented above, can be obtained from [13] and some additional remarks when applied to the axioms in [8]. It is important to understand how resolution with specifications works. In fact, a computed answer specification can be looked at as an extra condition or constraint (see also [7]) that needs to be satisfied before a given conclusion may be drawn from $P$. The completeness theorem simply states that from a logic program all such sufficient conditions can be generated. The completeness theorem leads almost directly to the following corollary.

Corollary 4.23. Let $A(\boldsymbol{x})$ be a atom and $\boldsymbol{u} \equiv u_{1}, \ldots, u_{k}$ complete terms, then:

$$
\boldsymbol{A}(\boldsymbol{u}) \in \mathrm{CS}_{P} \Leftrightarrow \text { for some } \rho(\boldsymbol{x}, \boldsymbol{y}) \text { and some } \boldsymbol{v}:(\boldsymbol{u}, \boldsymbol{v}) \in \rho \text { and } P \models \forall(\rho(\boldsymbol{x}, \boldsymbol{y}) \rightarrow A(\boldsymbol{x})) \text {. }
$$

\section{Finite failure}

In this section we will consider the negation as failure rule, for CSLD-resolution. It turns out that all "classical" results can be restored; even better: it seems that working in CSLD-semantics can simplify some theoretical constructions. Let us start with some definitions.

Definition 5.1. Let $G$ be a goal. A CSLD-tree for $P \cup\{G\}$ with c-rule $R$, is defined by

(i) every node of the tree is a goal and the root is equal to $G$

(ii) if $G^{\prime}=\left(\leftarrow A_{1}, \ldots, A_{m}, \ldots, A_{k}, \sigma\right)$ is a node for some consistent $\sigma$, and $R\left(G^{\prime}\right)=A_{m}$ then $G^{\prime}$ has a successor $\left(\leftarrow A_{1}, \ldots, A_{m-1}, B_{1}, \ldots, B_{q}, A_{m+1}, \ldots, A_{k}, \sigma \rho\right)$ 
for every clause $A \leftarrow B_{1}, \ldots, B_{q} \in P$, where $\rho \equiv \operatorname{mcu}\left(\left\{A_{m}, A\right\}\right)$; if $\sigma$ is inconsistent, then $G^{\prime}$ has no successor goals.

Definition 5.2. A success branch in a CSLD-tree is a branch that ends with $(\square, \sigma)$ for some consistent specification $\sigma$. A failure branch is a branch $\left(G_{i}, \sigma_{i}\right)_{0 \leqslant i \leqslant k}$ such that $\sigma_{k} \equiv \perp$.

Definition 5.3. A finitely failed CSLD-tree, or ff-tree for short, for $P \cup\{G\}$ is a finite CSLD-tree with only failure branches.

Definition 5.4. We will use the following notation:

$$
\begin{aligned}
& \mathrm{CT}_{P} \downarrow 0=\mathrm{CB}_{P}, \\
& \left.\mathrm{CT}_{P} \downarrow k+1=\mathrm{CT}_{P}\left(\mathrm{CT}_{P} \downarrow k\right)\right), \quad k \in \omega, \\
& \mathrm{CT}_{P} \downarrow \omega=\bigcap_{k=\omega} \mathrm{CT}_{P} \downarrow k .
\end{aligned}
$$

A well-known theorem says that $\mathrm{CT}_{P} \downarrow \omega$ is the greatest fixed point, denoted by $\operatorname{gfp}\left(\mathrm{CT}_{P}\right)$, of the continuous mapping $\mathrm{CT}_{P}$. Recall that $T_{P} \downarrow \omega$ does not need to be equal to the greatest fixed point of $T_{P}$.

Lemma 5.5. Let $A(x)$ be an atom and $\boldsymbol{u} \equiv u_{1}, \ldots, u_{k}$ be complete terms, then

$$
\begin{aligned}
A(\boldsymbol{u}) \in \mathrm{CT}_{P} \downarrow k+1 \Leftrightarrow & \text { there is a clause } A_{1}(\boldsymbol{y}) \leftarrow B_{1}(\boldsymbol{y}), \ldots, B_{q}(\boldsymbol{y}) \in P \text {, such } \\
& \text { that for some } \boldsymbol{v}:(\boldsymbol{u}, \boldsymbol{v}) \in \operatorname{mcu}\left(\left\{A(\boldsymbol{x}), A_{1}(\boldsymbol{y})\right\}\right) \text { and } \\
& B_{1}(\boldsymbol{v}), \ldots, B_{q}(\boldsymbol{v}) \in \mathrm{CT}_{P} \downarrow k .
\end{aligned}
$$

Theorem 5.6. Suppose $R$ is a fair c-rule, i.e. every atom in a goal clause is selected somewhere in any infinite derivation, and assume $\left(G_{i}, \sigma_{i}\right)_{i \in \omega}$ is an infinite CSLDderivation for $P \cup\left\{G_{0}, \sigma_{0}\right\}$ by $R$ with $G_{0}=\left(\leftarrow A_{1}(x), \ldots, A_{n}(\boldsymbol{x}), \sigma_{0}\right)$. If $\boldsymbol{u}$ are complete terms, then

$$
\forall k \exists i .\left((\boldsymbol{u}, \boldsymbol{v}) \in \sigma_{i}(\boldsymbol{x}, \boldsymbol{y}) \Rightarrow A_{1}(\boldsymbol{u}), \ldots, A_{n}(\boldsymbol{u}) \in \mathrm{CT}_{P} \downarrow k\right) .
$$

Proof. Let $k \in \omega$, and $\boldsymbol{u}$ complete terms. Let $\left(G_{i}, \sigma_{i}\right)_{i \in \omega}$ be an infinite derivation for $P \cup\left(G_{0}, \sigma_{0}\right)$. Then by induction on $k$ :

$(\boldsymbol{k}=\mathbf{0 )}$ : Immediately.

$(k+1)$ : Let $1 \leqslant j \leqslant n$ and let $m \in \omega$ such that $R\left(G_{m}\right)=A_{j}$. This $m$ exists because $R$ is fair. Then there is some clause $A(\boldsymbol{y}) \leftarrow B_{1}(\boldsymbol{y}), \ldots, B_{q}(\boldsymbol{y})$ such that $\rho(\boldsymbol{x}, \boldsymbol{y}) \equiv$ $\operatorname{mcu}\left(\left\{A_{j}(\boldsymbol{x}), A(\boldsymbol{y})\right\}\right) \quad$ is consistent and $\sigma_{m+1} \equiv \sigma_{m} \cdot \rho$. Assume $G_{m}=$ $\left(\leftarrow C_{1}, \ldots, A_{j}, \ldots, C_{r}\right)$ then $G_{m+1}=\left(\leftarrow C_{1}, \ldots, B_{1}, \ldots, B_{q}, \ldots, C_{r}\right)$ and clearly $\left(G_{m+i}, \sigma_{m+i}\right)_{i \geqslant 1}$ is an infinite derivation for $P \cup\left(G_{m+1}, \sigma_{m+1}\right)$. By induction, let $i^{\prime} \in \omega$ such that $(\boldsymbol{u}, \boldsymbol{v}) \in \sigma_{i^{\prime}}(\boldsymbol{x}, \boldsymbol{y}) \Rightarrow C_{1}(\boldsymbol{u}, \boldsymbol{v}), \ldots, B_{1}(\boldsymbol{v}), \ldots, B_{q}(\boldsymbol{v}), \ldots, C_{r}(\boldsymbol{u}, \boldsymbol{v}) \in \mathrm{CT}_{P} \downarrow k$, then it follows by definition of $\mathrm{CT}_{P}$ that for all $(\boldsymbol{u}, \boldsymbol{v}) \in \sigma_{i^{\prime}}(\boldsymbol{x}, \boldsymbol{y}) \Rightarrow A(\boldsymbol{v}) \in \mathrm{CT}_{P} \downarrow k+1$. Since $\sigma_{i^{\prime}} \leqslant \rho$ we have $\boldsymbol{A}_{j}(\boldsymbol{u}) \in \mathrm{CT}_{P} \downarrow k+1$. So, for every $j$ such an index $i^{\prime}$ exists. Now take the maximum of all $n$ indices. 
Lemma 5.7. If $(G, \sigma)$ has an ff-tree with depth $\leqslant k$, then $(G, \sigma \rho)$ has an ff-tree with depth $\leqslant k$.

Lemma 5.8. If for some ground specification $\sigma(x)$, the goal $\left(\leftarrow A_{1}(x), \ldots, A_{n}(x), \sigma(x)\right)$ has an ff-tree with depth $\leqslant k$, then for some $i \leqslant n,\left(\leftarrow A_{i}(x), \sigma(x)\right)$ has an ff-tree with depth $\leqslant k$.

Proof. By induction on $n$ :

$(n=1):$ Immediately.

$(n+1)$ : Assume $A_{n+1}(x)$ has no ff-tree with depth $\leqslant k$, then for all c-rules $R$, $P \cup\left\{A_{n+1}(x), \sigma(x)\right\}$ has a CSLD-derivation of length $\geqslant k+1$. Let $R$ be a c-rule such that $P \cup\left\{\leftarrow A_{1}(\boldsymbol{x}), \ldots, A_{n+1}(\boldsymbol{x}), \sigma(x)\right\}$ has an ff-tree with depth $\leqslant k$, and let $\left(G_{i}, \rho_{i}\left(\boldsymbol{x}, \boldsymbol{y}_{i}\right)\right)_{0 \leqslant i \leqslant k+1}$ be a derivation for $P \cup\left\{A_{n+1}(\boldsymbol{x}), \sigma(\boldsymbol{x})\right\}$ via $R$ with length $\geqslant k+1$, then for all derivations $\left(G_{i}^{\prime}, \tau_{i}\left(\boldsymbol{x}, \boldsymbol{z}_{i}\right)\right)_{0 \leqslant i \leqslant m}$ for $P \cup\left\{\leftarrow A_{1}(\boldsymbol{x}), \ldots, A_{n}(\boldsymbol{x}), \sigma(\boldsymbol{x})\right\}$ we have (by Propositions 4.2(iii) and 4.19) that for all $i, j, \tau_{i} \rho_{j} \equiv \perp \Rightarrow \tau_{i} \equiv \perp \vee \rho_{i} \equiv \perp$, since $\sigma(x)$ is ground and $\tau_{0}=\rho_{0}=\sigma$. Then, there is a finitely failed derivation $\left(G_{i}, \theta_{i}\right)_{0 \leqslant i \leqslant q}$ via $R$ for $P \cup\left\{\leftarrow A_{1}(\boldsymbol{x}), \ldots, A_{n+1}(\boldsymbol{x}), \sigma(\boldsymbol{x})\right\}$ such that $\theta_{i} \geqslant \tau_{i} \rho_{i}$ and $q \leqslant k$ (since all derivations via $R$ are finitely failed with length $\leqslant k$ ) we find that $\tau_{q} \rho_{q} \equiv \perp$. Because $\rho_{q}$ is consistent for all $q \leqslant k$ we have $\tau_{q} \equiv \perp$.

Therefore, all derivations for $P \cup\left\{\leftarrow A_{1}(x), \ldots, A_{n}(x), \sigma(x)\right\}$ are finitely failed with length $\leqslant k$, hence $P \cup\left\{\leftarrow A_{1}(x), \ldots, A_{n}(x), \sigma(x)\right\}$ has an ff-tree with depth $\leqslant k$. Now by induction.

Lemma 5.7 is easy to prove. Note that in Lemma 5.8, $\sigma$ needs to be ground (see also [12]).

Theorem 5.9. If $\boldsymbol{A}_{1}(\boldsymbol{u}), \ldots, \boldsymbol{A}_{n}(\boldsymbol{u}) \in \mathrm{CT}_{P} \downarrow k$ for some complete terms $\boldsymbol{u} \in \sigma(\boldsymbol{x})$, then there exists a ground specification $\rho(x, y) \leqslant \sigma(x)$ such that for $(\boldsymbol{v}, \boldsymbol{w}) \in \rho(\boldsymbol{x}, \boldsymbol{y})$, $A_{1}(v), \ldots, A_{n}(v) \in \mathrm{CT}_{P} \downarrow k$.

Proof. By induction on $k$. Assume $\boldsymbol{u} \in \sigma(\boldsymbol{x})$, for some specification $\sigma$.

$(\boldsymbol{k}=\mathbf{0})$ : Immediately, since any ground $\rho \leqslant \sigma$ suffices.

$(k+1)$ : Suppose $A_{1}(\boldsymbol{u}), \ldots, A_{n}(\boldsymbol{u}) \in \mathrm{CT}_{P} \downarrow k+1$ then by the definition of $\mathrm{CT}_{P}$ there exist clauses $A^{i}\left(\boldsymbol{y}_{i}\right) \leftarrow B_{1}^{i}\left(\boldsymbol{y}_{i}\right), \ldots, B_{q}^{i}\left(\boldsymbol{y}_{i}\right) \in P$ for all $i \leqslant n$, such that with $\rho^{i}\left(\boldsymbol{x}, \boldsymbol{y}_{i}\right) \equiv$ $\operatorname{mcu}\left(\left\{\boldsymbol{A}_{i}(\boldsymbol{x}), A^{i}\left(y_{i}\right)\right\}\right), \rho=\rho^{1} \cdots \rho^{n}$ is consistent. Clearly, $\sigma \rho\left(\boldsymbol{x}, \boldsymbol{y}_{1}, \ldots, \boldsymbol{y}_{n}\right)$ is consistent as well. Writing $\boldsymbol{y} \equiv \boldsymbol{y}_{1}, \ldots, y_{n}$ there exist $\boldsymbol{v} \equiv \boldsymbol{v}_{1}, \ldots, \boldsymbol{v}_{n}$ such that $(\boldsymbol{u}, \boldsymbol{v}) \in$ $\sigma \rho(\boldsymbol{x}, \boldsymbol{y})$ and for all $i \leqslant n: B_{1}^{i}\left(\boldsymbol{v}_{i}\right), \ldots, B_{q}^{i}\left(\boldsymbol{v}_{i}\right) \in \mathrm{CT}_{P} \downarrow k$. It follows by induction that there exists a ground specification $\tau(\boldsymbol{x}, \boldsymbol{y}, \boldsymbol{z}) \leqslant \sigma \rho(\boldsymbol{x}, \boldsymbol{y})$ such that for $\left(\boldsymbol{w}, \boldsymbol{w}^{\prime}, \boldsymbol{w}^{\prime \prime}\right) \in$ $\tau(\boldsymbol{x}, \boldsymbol{y}): B_{1}^{i}\left(\boldsymbol{w}^{\prime}\right), \ldots, B_{q}^{i}\left(\boldsymbol{w}^{\prime}\right) \in \mathrm{CT}_{P} \downarrow k$. Since $\rho \leqslant \rho^{i}$ we have $A_{i}(\boldsymbol{w}) \equiv A^{i}\left(\boldsymbol{w}^{\prime}\right)$ and therefore $A_{1}(w), \ldots, A_{k}(w) \in \mathrm{CT}_{P} \downarrow k+1$, by definition of $\mathrm{CT}_{P}$. Since $\sigma \rho \leqslant \sigma$ we find $\tau \leqslant \sigma \rho \leqslant \sigma$.

Theorem 5.10. If $\sigma(x)$ is ground and $\left(\leftarrow A_{1}(x), \ldots, A_{n}(x), \sigma(x)\right)$ has an ff-tree with depth $\leqslant k$, then for some $i \leqslant n$

$$
\forall u .\left(u \in \sigma(x) \Rightarrow A_{i}(u) \notin C T_{P} \downarrow k\right) .
$$


Proof. By induction on $k$.

$(k=1)$ : Directly by Lemma 5.5 .

$(\boldsymbol{k}+1)$ : Suppose $A_{i}(\boldsymbol{u}) \in \mathrm{CT}_{P} \downarrow k+1$, for $\boldsymbol{u} \in \sigma$, then there is a clause $A(\boldsymbol{y}) \leftarrow$ $B_{1}(\boldsymbol{y}), \ldots, B_{q}(\boldsymbol{y}) \in P$ such that $\rho(\boldsymbol{x}, \boldsymbol{y}) \equiv \operatorname{mcu}\left(\left\{A_{i}(\boldsymbol{x}), A(\boldsymbol{y})\right\}\right)$ is consistent and for some $\boldsymbol{v}:(\boldsymbol{u}, \boldsymbol{v}) \in \rho$ and $B_{1}(\boldsymbol{v}), \ldots, B_{q}(\boldsymbol{v}) \in \mathrm{CT}_{P} \downarrow k$ (see Lemma 5.5). Clearly $\sigma \rho$ is consistent and $(\boldsymbol{u}, \boldsymbol{v}) \in \sigma \rho(\boldsymbol{x}, \boldsymbol{y})$. Then, by Theorem 5.9 it follows that there is some ground specification $\tau(\boldsymbol{x}, \boldsymbol{y}, \boldsymbol{z}) \leqslant \sigma \rho(\boldsymbol{x}, \boldsymbol{y})$ such that: $B_{1}\left(\boldsymbol{v}^{\prime}\right), \ldots, B_{q}\left(\boldsymbol{v}^{\prime}\right) \in \mathrm{CT}_{P} \downarrow k$ for all $\left(\boldsymbol{u}^{\prime}, \boldsymbol{v}^{\prime}, \boldsymbol{w}^{\prime}\right) \in \tau$. Since $\tau$ is ground, it follows by induction that $\left(\leftarrow B_{1}(\boldsymbol{y}), \ldots, B_{q}(\boldsymbol{y})\right.$, $\tau(x, y, z))$ has no ff-tree with depth $\leqslant k$, hence $P \cup\left(\leftarrow A_{i}(x), \tau(x, y, z)\right)$ has no ff-tree with depth $\leqslant k+1$. Since $\tau \leqslant \sigma$ and both $\tau$ and $\sigma$ are ground, it follows that $\tau \equiv_{x} \sigma$. Hence $P \cup\left(\leftarrow A_{i}(x), \sigma(x)\right)$ has no ff-tree with depth $\leqslant k+1$.

Thus, we have proved that if $A_{i}(\boldsymbol{u}) \in \mathrm{CT}_{P} \downarrow k+1$, then $P \cup\left(\leftarrow A_{i}(\boldsymbol{x}), \sigma\right)$ has no ff-tree with depth $\leqslant k+1$. Suppose for all $1 \leqslant i \leqslant n, P \cup\left(\leftarrow A_{i}(x), \sigma\right)$ has no ff-tree with depth $\leqslant k+1$, then it follows by Lemma 5.8 that $P \cup\left(\leftarrow A_{1}(x), \ldots, A_{n}(x), \sigma(x)\right)$ has no such ff-tree.

Corollary 5.11. If $(\leftarrow A(x), \sigma(x))$ has an ff-tree with depth $\leqslant k$, then

$$
\forall \boldsymbol{u} .\left(\boldsymbol{u} \in \sigma(\boldsymbol{x}) \Rightarrow A(\boldsymbol{u}) \notin \mathrm{CT}_{P} \downarrow k\right) .
$$

Note, that in Corollary 5.11, $\sigma(\boldsymbol{x})$ does not need to be ground. Its proof follows from the last three lines of the proof of Theorem 5.10.

Clearly, from a program one cannot derive any negative formulas. As usual, we can consider negation as finite failure of the computation. Consider the following definition.

Definition 5.12. The complete failure set, $\mathrm{CF}_{P}$, of a program $P$ is defined by

$$
\begin{gathered}
\mathrm{CF}_{P}=\left\{\boldsymbol{A}(\boldsymbol{u}) \in \mathrm{CB}_{P}:\right. \\
\text { there is a specification } \sigma(\boldsymbol{x}) \text { such that } \boldsymbol{u} \in \sigma \\
\text { and }(\leftarrow \boldsymbol{A}(\boldsymbol{x}), \sigma(\boldsymbol{x})) \text { has an ff-tree }\}
\end{gathered}
$$

Proposition 5.13. $\mathrm{CF}_{P}$ is well-defined.

Proof. Assume $A(\boldsymbol{u}) \in \mathrm{CF}_{P}$ and suppose $d\left(A(\boldsymbol{u}), A_{1}(\boldsymbol{v})\right)=0$. Let $\boldsymbol{u} \in \sigma(\boldsymbol{x})$ such that $(\leftarrow A(x), \sigma(x))$ has an ff-tree. Since $\rho(x, y) \equiv \operatorname{mcu}\left(\left\{A(x), A_{1}(y)\right\}\right)$ is consistent, and $(\boldsymbol{u}, \boldsymbol{v}) \in \rho$ we find that $\sigma \rho(\boldsymbol{x}, \boldsymbol{y})$ is consistent. Now it is easy to see that any derivation for $P \cup(\leftarrow A(x), \sigma(x))$ corresponds to a derivation for $P \cup\left(\leftarrow A_{1}(y), \sigma \rho(x, y)\right)$, hence $P \cup\left(\leftarrow A_{1}(y), \sigma \rho(x, y)\right)$ has an ff-tree. Since $(u, v) \in \sigma \rho(x, y)$, we find $A_{1}(v) \in$ $\mathrm{CF}_{P}$.

Proposition 5.14. Suppose $\sigma_{1}(\boldsymbol{x}), \ldots, \sigma_{k}(\boldsymbol{x})$ are specifications and $\boldsymbol{u}$ are complete terms such that $\boldsymbol{u} \notin \sigma_{1}(\boldsymbol{x}), \ldots, \boldsymbol{u} \notin \sigma_{k}(\boldsymbol{x})$, then there exists a consistent specification $\rho(\boldsymbol{x})$ such that $\boldsymbol{u} \in \rho(\boldsymbol{x})$ and for all $1 \leqslant i \leqslant k, \rho \sigma_{i}(\boldsymbol{x}) \equiv \perp$. 
Theorem 5.15. $\mathrm{CF}_{P}=\mathrm{CB}_{P} / \mathrm{CT}_{P} \downarrow \omega$.

Proof. ( $\subseteq$ ): Suppose $A(\boldsymbol{u}) \in \mathrm{CF}_{P}$ then there exists a specification $\sigma(\boldsymbol{x})$, such that $\boldsymbol{u} \in \sigma$ and $P \cup(\leftarrow A(x), \sigma)$ has an ff-tree with depth $\leqslant k$, say. By Corollary 5.11 it follows that $A(\boldsymbol{u}) \notin \mathrm{CT}_{P} \downarrow k$, hence $A(\boldsymbol{u}) \notin \mathrm{CT}_{P} \downarrow \omega$.

(): Suppose $A(\boldsymbol{u}) \notin \mathrm{CT}_{P} \downarrow \omega$ then $A(\boldsymbol{u}) \notin \mathrm{CT}_{P} \downarrow k$, for some $k \in \omega$, and so by Lemma 5.6 there is no infinite fair derivation $\left(G_{i}\left(x, y_{i}\right), \rho_{i}\left(x, y_{i}\right)\right)_{i \in \omega}$ for $P \cup$ $\{\leftarrow A(x), \emptyset\}$, such that for all $i: \exists v_{i} .\left(u, v_{i}\right) \in \rho_{i}$. Now, let $R$ be a fair c-rule and let $T$ be the CSLD-tree for $P \cup(\leftarrow A(x), \emptyset)$ with c-rule $R$. Delete from $T$ all successors of nodes $(G(x, y), \sigma(x, y))$ for which for all $\boldsymbol{v}$ : $(\boldsymbol{u}, \boldsymbol{v}) \notin \sigma$. Clearly, the remaining tree $T^{\prime}$ is finite, since otherwise there would be an infinite derivation as mentioned above. Moreover, $T$ cannot contain any success branches with last node $(\square, \theta)$ such that for some $v:(\boldsymbol{u}, \boldsymbol{v}) \in \theta$; otherwise by Theorem 4.17, $A(\boldsymbol{u}) \in \mathrm{CT}_{P} \downarrow \omega$ which is impossible since $\mathrm{CT}_{P} \uparrow \omega \subseteq \mathrm{CT}_{P} \downarrow k$. Therefore in all the leaves $\left(G^{\prime}(\boldsymbol{x}, \boldsymbol{y}), \theta^{\prime}(\boldsymbol{x}, \boldsymbol{y})\right)$ of $T^{\prime}$, we have that for all $v$ : $(\boldsymbol{u}, \boldsymbol{v}) \notin \theta^{\prime}$. Since $T^{\prime}$ has only finitely many leaves, by Proposition 5.14, there exists a specification $\rho(\boldsymbol{x}, \boldsymbol{y})$ such that for some $\boldsymbol{v}:(\boldsymbol{u}, \boldsymbol{v}) \in \rho$ and for all leaves $\left(G^{\prime}(\boldsymbol{x}, \boldsymbol{y}), \theta^{\prime}(\boldsymbol{x}, \boldsymbol{y})\right)$ of $T^{\prime}, \rho \theta^{\prime} \equiv \perp$. Thus, $(\leftarrow \boldsymbol{A}(\boldsymbol{x}), \rho(\boldsymbol{x}, y))$ has an ff-tree and for some $\boldsymbol{v}:(\boldsymbol{u}, \boldsymbol{v}) \in \rho$.

Theorem 5.15 can be considered as a corollary from results in [10]. It turns out that programs with CSLD-resolution have the nice property that the greatest fixed point of $\mathrm{CT}_{P}$ is precisely the set of all non-failing (complete) atoms. Furthermore we found, that the complete success set is $\operatorname{If}\left(\mathrm{CT}_{P}\right)$, so both sets can be described in terms of fixed points of $\mathrm{CT}_{p}$. Therefore we have reason to believe that CSLDsemantics for logic programs may have a few nice properties extra that do not exist in "classical" SLD-semantics.

Definition 5.16. The completion, $\operatorname{comp}(P)$, of a program $P$ consists of the equational theory $\operatorname{Eq}(P)$, together with the set of all formulas

$$
\begin{aligned}
\forall x . p(x) \leftrightarrow & \left(\exists y . \sigma_{1}(x, y) \wedge B_{1}^{1}(y) \wedge \cdots \wedge B_{k}^{1}(y)\right) \vee \cdots \\
& \vee\left(\exists y . \sigma_{n}(x, y) \wedge B_{1}^{n}(y) \wedge \cdots \wedge B_{k}^{n}(y)\right)
\end{aligned}
$$

corresponding to the collection of all clauses

$$
A_{1}(y) \leftarrow B_{1}^{1}(y), \ldots, B_{k}^{1}(y), \ldots, A_{n}(y) \leftarrow B_{1}^{n}(y), \ldots, B_{k}^{n}(y)
$$

in $P$, such that $\sigma_{i}(\boldsymbol{x}, \boldsymbol{y})=\operatorname{mcu}\left(\left\{p(\boldsymbol{x}), A_{i}(\boldsymbol{y})\right\}\right)$ and $p$ is a predicate symbol.

Theorem 5.17 (soundness of the negation as failure rule)

$$
\left(\leftarrow A_{1}(\boldsymbol{x}), \ldots, A_{n}(\boldsymbol{x}), \sigma\right) \text { has an ff-tree } \Rightarrow \operatorname{comp}(P) \models \forall\left(\sigma \rightarrow \neg\left(A_{1} \wedge \cdots \wedge A_{n}\right)\right) .
$$

Proof. Suppose $\left(\leftarrow A_{1}(\boldsymbol{x}), \ldots, A_{n}(\boldsymbol{x}), \boldsymbol{\sigma}(\boldsymbol{x})\right)$ has an ff-tree with depth $\leqslant k$ via c-rule $R$, say. Now by induction on $k$. 
$(\boldsymbol{k}=1)$ : Suppose $R\left(\leftarrow A_{1}(\boldsymbol{x}), \ldots, A_{n}(\boldsymbol{x})\right)=A_{i}(x), \quad P$ has clauses $C_{j}\left(\boldsymbol{y}_{j}\right) \leftarrow$ $B_{1}^{j}\left(\boldsymbol{y}_{j}\right), \ldots, B_{q}^{j}\left(\boldsymbol{y}_{j}\right)$ and define $\rho_{j}\left(\boldsymbol{x}, \boldsymbol{y}_{j}\right)=\operatorname{mcu}\left(\left\{A_{i}(\boldsymbol{x}), C_{j}\left(\boldsymbol{y}_{j}\right)\right\}\right)$. Clearly for all $j \leqslant n$, $\sigma \rho_{j}\left(\boldsymbol{x}, \boldsymbol{y}_{j}\right)$ is inconsistent and hence $\mathrm{Eq}(P) \models \forall\left(\sigma \rightarrow \neg \rho_{j}\right)$ for all $j$. Therefore $\operatorname{comp}(P) \models \forall\left(\sigma \rightarrow \neg A_{i}\right)$ and hence $\operatorname{comp}(P) \vDash \forall\left(\sigma \rightarrow \neg\left(A_{1} \wedge \cdots \wedge A_{n}\right)\right)$.

$(\boldsymbol{k}+1)$ : Suppose for some $j, \sigma \rho_{j}\left(\boldsymbol{x}, \boldsymbol{y}_{j}\right)$ is consistent. Then, for every such index $j$ it follows that

$$
\left(\leftarrow A_{1}(\boldsymbol{x}), \ldots, A_{i-1}(\boldsymbol{x}), B_{1}^{j}\left(y_{j}\right), \ldots, B_{q}^{j}\left(y_{j}\right), A_{i+1}(\boldsymbol{x}), \ldots, A_{n}(\boldsymbol{x}), \sigma \rho_{j}\left(\boldsymbol{x}, \boldsymbol{y}_{j}\right)\right)
$$

has an ff-tree with depth $\leqslant k$. Hence for all $j \leqslant n$,

$$
\operatorname{comp}(P) \vDash \forall\left(\sigma \rho_{j} \rightarrow \neg\left(A_{1} \wedge \cdots \wedge A_{i-1} \wedge B_{1}^{j} \wedge \cdots \wedge B_{q}^{j} \wedge A_{i+1} \wedge \cdots \wedge A_{n}\right)\right) .
$$

Since

$$
\operatorname{comp}(P) \vDash \forall \boldsymbol{x} .\left(A_{i}(\boldsymbol{x}) \leftrightarrow \exists \boldsymbol{y}_{1} .\left(\rho_{1} \wedge B_{1}^{1} \wedge \cdots \wedge B_{q}^{1}\right) \vee \cdots \vee \exists \boldsymbol{y}_{n} .\left(\rho_{n} \wedge B_{1}^{n} \wedge \cdots \wedge B_{q}^{n}\right)\right)
$$

it easily follows that $\operatorname{comp}(P) \vDash \forall\left(\sigma \rightarrow \neg\left(A_{1} \wedge \cdots \wedge A_{n}\right)\right)$, for all $j \leqslant n$.

Theorem 5.17 is a strengthening of a similar theorem from [8] by weakening the equality theory.

Lemma 5.18. $M \subseteq \mathrm{CB}_{P}$ is a model for $\operatorname{comp}(P)$ if and only if $\mathcal{M}$ is a fixed point of $\mathrm{CT}_{P}$.

Theorem 5.19 (completeness of the negation as failure rule). Suppose $R$ is a fair computation rule, i.e. every subgoal is selected in a finite number of steps. Then $\operatorname{comp}(P) \models \forall\left(\sigma \rightarrow \neg\left(A_{1} \wedge \cdots \wedge A_{n}\right)\right) \Rightarrow P \cup\left(\leftarrow A_{1}(\boldsymbol{x}), \ldots, A_{n}(\boldsymbol{x}), \sigma\right)$ has an ff-tree via $R$.

Proof. Suppose $\left(\leftarrow A_{1}(\boldsymbol{x}), \ldots, A_{n}(\boldsymbol{x}), \sigma_{0}\right)$ does not have a finitely failed CSLD-tree. It is proved that $\operatorname{gfp}\left(\mathrm{CT}_{P}\right) \models \operatorname{comp}(P) \cup\left\{\exists\left(A_{1} \wedge \cdots \wedge A_{n} \wedge \sigma_{0}\right)\right\}$. Let $R$ be a fair computation rule. Suppose $\left(G_{i}, \sigma_{i}\left(\boldsymbol{x}, \boldsymbol{y}_{i}\right)\right)_{i \in \omega}$ is a non-failed CSLD-derivation for $P \cup\left(\leftarrow A_{1}(x), \ldots, A_{n}(x), \sigma_{0}\right)$ via $R$. Define $\sum\left(x, y_{1}, y_{2}, \ldots\right)=\bigcup_{i \in \omega} \sigma_{i}\left(x, y_{i}\right)$. Since $\sum \cup \operatorname{Eq}(P)$ is finitely satisfiable it follows from the compactness theorem that $\sum \cup \operatorname{Eq}(P)$ has a model. Then, by Theorem $3.16, \sum$ is satisfiable in every complete Herbrand model and hence $\operatorname{gfp}\left(\mathrm{CT}_{P}\right) \cup \sum$ is satisfiable. Let $\boldsymbol{u}$ be such that $\sum[\boldsymbol{x} / \boldsymbol{u}]$ is satisfiable in $\operatorname{gfp}\left(\mathrm{CT}_{P}\right)$. Then for all $i, \sigma_{i}[\boldsymbol{x} / \boldsymbol{u}]$ is satisfiable, and hence there exist $\boldsymbol{v}_{i}$ such that for all $i,\left(\boldsymbol{u}, \boldsymbol{v}_{i}\right) \in \sigma_{i}\left(\boldsymbol{x}, \boldsymbol{y}_{i}\right)$. Especially we find that $\boldsymbol{u} \in \sigma_{0}(\boldsymbol{x})$. Since $R$ is fair it follows from Theorem 5.6 that $A_{1}(\boldsymbol{u}), \ldots, A_{n}(\boldsymbol{u}) \in \mathrm{CT}_{P} \downarrow k$ for all $k$, hence $\boldsymbol{A}_{1}(\boldsymbol{u}), \ldots, A_{n}(\boldsymbol{u}) \in \mathrm{CT}_{P} \downarrow \omega$ and therefore $A_{1}(\boldsymbol{u}), \ldots, A_{n}(\boldsymbol{u}) \in \operatorname{gfp}\left(\mathrm{CT}_{P}\right)$, since $\mathrm{CT}_{P} \downarrow \omega=\operatorname{gfp}\left(\mathrm{CT}_{P}\right)$. By Lemma 5.18 we have $\operatorname{gfp}\left(\mathrm{CT}_{P}\right) \vDash \operatorname{comp}(P)$, so $\operatorname{gfp}\left(\mathrm{CT}_{P}\right) \vDash \operatorname{comp}(P) \cup\left\{\exists\left(A_{1} \wedge \cdots \wedge A_{n} \wedge \sigma_{0}\right)\right\}$.

Theorem 5.19 is an immediate consequence of the corresponding theorem in [8]. Its proof is much simpler than the proof in [12] for SLD-resolution, due to the fact that the model $\operatorname{gfp}\left(\mathrm{CT}_{P}\right)$ is a complete Herbrand model. In the case of SLDresolution, a completeness theorem for negation as failure was proved in [9] and 
[15] by constructing a model for $\operatorname{comp}(P) \cup\left\{\exists\left(A_{1} \wedge \cdots \wedge A_{n} \wedge \sigma_{0}\right)\right\}$ which is not a Herbrand model. This serious complication in the proof is overcome by the fact that $\mathrm{CT}_{P} \downarrow \omega=\operatorname{gfp}\left(\mathrm{CT}_{P}\right)$ (for other interpretations of fixed points see for instance [11]).

We have the following corollary which cannot be obtained in ordinary SLD. semantics.

Corollary 5.20. $\operatorname{comp}(P) \cup\{A \wedge \sigma\}$ has a complete Herbrand model iff it has a model.

\section{Acknowledgment}

I especially want to thank Krzysztof Apt for his clarifying comments, Jos Baeten for pointing out some errors in a draft version and Johan van Benthem for giving his support to this paper.

\section{References}

[1] K.R. Apt and M.H. van Emden, Contributions to the theory of logic programming, J. Assoc. Comput. Mach. 29 (1982) 841-862.

[2] A. Colmerauer, Prolog and infinite trees, in: Clark and Tarnlund, eds., Logic Programming, (Academic Press, New York, 1982) 231-251.

[3] A. Colmerauer, Prolog II Reference Manual and Theoretical Model (Groupe Intelligence Artificielle, Faculté des Sciences de Luming, Marseille, 1982).

[4] B. Courcelle, Fundamental properties of infinite trees, Theoret. Comput. Sci. 25 (1983) 95-169.

[5] M.H. van Emden and R.A. Kowalski, The semantics of predicate logic as a programming language, J. Assoc. Comput. Mach. 23 (1976) 733-742.

[6] M.H. van Emden and J.W. Lloyd, A logical reconstruction of Prolog II, J. Logic Programming 1 (1984) $143-150$

[7] J. Jaffar and L.-L. Lassez, Constraint logic programming, in: Proc. POPL, Munich (1987) 111-119.

[8] J. Jaffar, J-L. Lassez and M.J. Maher, Prolog II as an instance of the logic programming language scheme, in: M. Wirsing, ed., Proc. IFIP Conf. (North-Holland, Amsterdam, 1987).

[9] J. Jaffar, J-L. Lassez and J.W. Lloyd, Completeness of the negation as finite failure rule, in: Proc. IJCAI-83, Karlsruhe (1983) 500-506.

[10] J. Jaffar and P. Stuckey, Semantics of infinite tree logic programming, Theoret. Comput. Sci. 46 (1986) 141-158.

[11] G. Levi and C. Palamidessi, Contributions to the semantics of logic perpetual processes, Draft manuscript, Dipartimento di Informatica, Università di Pisa, Italy, 1987.

[12] J.W. Lloyd, Foundations of Logic Programming (Springer-Verlag, Heidelberg, 1984).

[13] M.J. Maher, Logic semantics for a class of committed-choice programs, in: Proc. 4th. Int. Conf. on Logic Programming, Melbourne (1987) 858-876.

[14] D.A. Plaisted, The occur-check problem in Prolog, in: Proc. Int. Symp. on Logic Programming, Atlantic City (1984) 272-280.

[15] D.A. Wolfram, M.J. Maher and J.-L. Lassez, A unified treatment of resolution strategies for logic programs, in: Proc. Second Int. Logic Programming Conf., Uppsala (1984) 263-276. 